\title{
3 Research Square \\ Potential Target Screening and Molecular Docking of Newly Isolated Compound From Inonotus Obliquus
}

\section{Zhen-Dong Liu}

Key Laboratory of Saline-alkali Vegetation Ecology Restoration, Ministry of Education, College of Life Sciences, Northeast Forestry University

Yi Liu

Food Science College, Tibet Agriculture \& Animal Husbandry University

\section{Liang Li}

Food Science College, Tibet Agriculture \& Animal Husbandry University

\section{Bei Xue}

Food Science College, Tibet Agriculture \& Animal Husbandry University

\section{Wenhan Wang ( $\square$ wangwenhan@saas.sh.cn )}

Institute of Edible Fungi, Shanghai Academy of Agricultural Sciences, Key Laboratory of Edible Fungi Resources and Utilization (South), Ministry of Agriculture

\section{Xiu-Feng Yan}

College of Life and Environmental Science, Wenzhou University, Chashan University Town

\section{Research Article}

Keywords: Inonotus obliquus, Virtual screening, Target prediction, Molecular docking, Pharmacophore

Posted Date: February 26th, 2021

DOl: https://doi.org/10.21203/rs.3.rs-250772/v1

License: (c) (1) This work is licensed under a Creative Commons Attribution 4.0 International License. Read Full License 


\section{Abstract}

Compound (1-(4-hydroxyphenyl)-3-methoxypropan-1-one) is a newly isolated compound from Inonotus obliquus. In this paper, four target protein prediction methods were used to search the potential targets of compounds. The target proteins were determined according to the corresponding screening criteria. The target proteins with therapeutic value were docked with compound and target proproteinogen ligands by molecular docking software, and their binding affinity with target proteins was compared. The pharmacophore analysis program was used to investigate whether the pharmacophore characteristics of the compound and the original ligand compounds were consistent. 26 target proteins were obtained by virtual screening. The PASS server filtered out 2 targets. 7 targets were screened by the PharmMapper database. 2 targets were obtained by screening the PDB protein database. 18 targets were obtained by ROCS screening. Compared with the binding affinity of molecular docking, it was found that the binding affinity of the compound to oxysterols receptor LXR-beta, chymase, heat shock protein HSP 90-alpha, carbonic anhydrase 2 and arylamine $\mathrm{N}$-acetyltransferase Nat was higher than that of the original ligands. It shows that the compound has potential applications in anti-tumor, anti-inflammatory, affecting intestinal flora and affecting human lipid transport. Pharmacophore analysis was performed using LigandScout software. The results of pharmacophore analysis show that the phenolic hydroxyl group in the compound is the key group and can participate in the interaction with amino acids. Besides, the mother nuclear benzene ring can also participate in the hydrophobic interaction.

\section{Introduction}

Natural products are an important source of new drug creation and are often used in the field of drug discovery and design. Drug targets are molecules in which drugs play a role to achieve a curative effect, which can combine with drug molecules and produce pharmacological effects. Drug target is not only the basis of drug screening, but the premise of innovative drugs, which is of source innovation significance for the creation of new drugs. Identifying drug targets can explore the applicability of drugs and provide possible new strategies for clinical prevention and treatment. The reverse target search can be carried out with the compound as the target by using the computer virtual screening technology ${ }^{1,2}$. This overcomes the deficiency that the traditional drug target screening methods can only study on a single target. Thus, the action targets of natural products can be found more efficiently.

Inonotus obliquus is a kind of wood-rot fungus that grows in the cold zone. People usually use this fungus to prevent and cure all kinds of difficult and complicated diseases. Inonotus obliquus is a medicinal fungus with a variety of biological activities, including anti-tumor ${ }^{3,4}$, hypoglycemic ${ }^{5}$, antiinflammatory ${ }^{6}$, anti-virus, and enhance immunity and other biological activities ${ }^{7}$. This fungus has no obvious physiological toxicity. In recent years, researchers have continuously studied the chemical constituents and physiological activities of Inonotus obliquus. The research reports on the chemical constituents and pharmacological activities of Inonotus obliquus are also increasing. Inonotus obliquus contains a variety of chemical constituents ${ }^{8}$, the main compounds are triterpenes ${ }^{9}$, polysaccharides ${ }^{10}$, 
and sesquiterpenes ${ }^{11}$. A new compound (1-(4-hydroxyphenyl)-3-methoxypropan-1-one) (Fig. 1 ) isolated from Inonotus obliquus was studied in this paper. The virtual screening of the target of the compound was carried out by using the PASS server, PharmMapper database, PDB database, and ROCS. The binding affinity of compounds to potential targets was further investigated by molecular docking software for the main target proteins with therapeutic value for human diseases. This provides theoretical guidance and scientific experimental basis for the discovery of potential drug targets of compounds.

\section{Materials And Methods}

\section{Preparation of compound and proteins}

Compound preparation; The 2D structure of the compound was drawn in Marvin Sketch software, and the 2D structure was transformed into the 3D structure. Save the treated compound in *.mol2 format. Import the *.Mol2 file of the compound into the Ligprep module in the Schrodinger package for ligand preparation, that is, ionization $(\mathrm{pH}: 7.0+/-2.0)$, desalination, generation of tautomers, etc. The ligand was optimized using the same force field (OPLS $₫ 2005$ ) as in the receptor optimization process. Other parameters were software default values.

Protein preparation; according to the target information obtained by virtual screening, the protein structures were downloaded from the PDB protein database (https://www.rcsb.org/)) for molecular docking.

\section{Target screening of compound}

Target screening using the PASS server, The PASS (http://www.pharmaexpert.ru/passonline/) server can predict the potential targets of drug-like compounds based on small molecular active fragments ${ }^{12}$. We used the PASS online server to predict the different biological activities of the compound according to the compound's structure. When the constructed compound 2D mol format is entered into PASS's SAR training set database, the server will automatically represent the structure in the form of MNA (Multilevel Neighborhoods of Atoms) descriptors. The prediction program will match the compound with the training set to get a list of biological activities. The target with prediction accuracy $\mathrm{Pa}>0.9$ was selected as the possible active target of the compound. ${ }^{13}$ All parameters in the calculation process are set by system default.

Target prediction in the PharmMapper database; The PharmMapper (http://59.78.96.61/pharmmapper/submit_file.php) server was used to predict the target of the compound ${ }^{13}$. The server can predict the target in a few minutes to tens of minutes. When the mol format file of the compound is uploaded to the PharmMapper web server, the server can automatically find the pharmacophore that best matches the molecular conformation of the query, and sort it according to its matching program ${ }^{14}$. The server can provide protein targets of the top 300 points. The score represents the degree of agreement between the small molecule and the pharmacophore model. The higher the 
score, the more likely it is to be a target protein for drug molecules. We determine the potential targets of the compound based on the Normalized Fit Score.

Target prediction in the PDB database; the PDB protein database (http://www.rcsb.org/pdb/ligand/chemAdvSearch.do), which provides a lot of information about proteins and ligands, is the most important database for collecting biological macromolecular structures. The target of the compound can be predicted by searching for ligands with a similar structure of the compound.

Target prediction using ROCS; ROCS (http://targetfishing.molcalx.com.cn/) is a program developed by OpenEye to compare the shape and pharmacophore similarity of compounds ${ }^{15}$. ROCS can be used to predict the multi-classification targets of compounds ${ }^{16}$. It has been proved that ROCS can be successfully used for target prediction ${ }^{17,18}$. We use ROCS to search the database of known active compounds to predict the targets of the compound in Alzheimer's disease, cancer, diabetes, and, inflammation. The ShapeTanimoto value was used to evaluate the screened targets.

\section{Molecular docking and Pharmacophore model construction}

Molecular docking; iGEMDOCK (http://gemdock.life.nctu.edu.tw/dock/igemdock.php) is an open-source molecular docking software that can dock compound molecules to the receptor protein binding pocket ${ }^{19}$. To investigate the binding activity of the compound to the target protein, the molecular docking of the compound was studied by using the computer-aided drug design software package iGEMDOCK. The crystal structure of the target protein for molecular docking was downloaded by searching the protein target library. In the study, the original ligand in the receptor was used as the positive control (reference compound). The active sites are determined by the coordinates of the co-crystalline compounds of each protein. In molecular docking, the groups on the protein can bind to the compound molecule through van der Waals force, hydrogen bond, and electrostatic interaction to match the binding site with the lowest energy. The binding degree of the target protein and the compound is judged by the binding energy. When the binding conception of the compound and the receptor is stable, the energy is lower, and the greater the possibility of interaction is.

Pharmacophore model construction; The pharmacophore model can be constructed according to the receptor-ligand interaction model by LigandScout software ${ }^{20}$. Based on the results of molecular docking calculation, the action mode of the compound was analyzed by the Ligandscout program to determine whether the target compound matched the pharmacophore characteristics of the target protein.

\section{Results}

\section{PASS server target filtering results}

We used the PASS server to predict the target of the compound. Biological targets are identified based on $\mathrm{Pa}$ close to 1 and $\mathrm{Pi}$ close to $\mathrm{O}$. Among the screening results, target proteins with $\mathrm{Pa}>0.9$ and related to 
human diseases were selected for analysis. Two potential targets were obtained by virtual screening, namely membrane integrity agonist and chlordecone reductase inhibitor. Target analysis showed that the compound may be a potential membrane integrity agonist and chlordecone reductase inhibitor.

Therefore, the compound may have potential application value in diabetes and inflammation. The details are shown in Table 1.

\section{PharmMapper database target screening results}

The PharmMapper server is used to predict the target of the compound by reverse matching method. According to the Normalized Fit Score score, protein targets with Normalized Fit Score $>0.8$ and related to human diseases were screened for further analysis. 7 valuable targets were obtained from 300 target information. The main therapeutic targets are phospholipase A2, acetylcholinesterase, arylamine $\mathrm{N}$ acetyltransferase, peptidyl-prolyl cis-trans isomerase $\mathrm{A}$, chymase, oxysterols receptor $\mathrm{LXR}$-beta, triggering receptor expressed on myeloid cells 1 . These target proteins are involved in human diseases including Alzheimer's disease, cancer, cardiovascular disease, infectious diseases, etc., The result shows that the newly isolated compounds from Inonotus obliquus have wide application potential. The target information is shown in Table 2.

Table 1

Prediction result of PASS

\begin{tabular}{|llll|}
\hline Rank & Pa & Pi & Targets \\
\hline 1 & 0,922 & 0,006 & Membrane integrity agonist \\
\hline 2 & 0,903 & 0,005 & Chlordecone reductase inhibitor \\
\hline \multicolumn{4}{|l}{ Pa: Probability of activity. Pi: Probability of inactivity. } \\
\hline
\end{tabular}

Table 2

Target prediction results of PharmMapper

\begin{tabular}{|lllll|}
\hline Rank & $\begin{array}{l}\text { PDB } \\
\text { ID }\end{array}$ & Target Name & $\begin{array}{l}\text { Normalized Fit } \\
\text { Score }\end{array}$ & $\begin{array}{l}\text { Number of } \\
\text { Features }\end{array}$ \\
\hline 1 & 1SV3 & Phospholipase A2 & 0.9896 & 3 \\
\hline 2 & 1DX6 & Acetylcholinesterase & 0.9873 & 3 \\
\hline 3 & 1W6F & Arylamine N-acetyltransferase & 0.9566 & 3 \\
\hline 4 & 1W8M & Peptidyl-prolyl cis-trans isomerase A & 0.9468 & 3 \\
\hline 5 & 1KLT & Chymase & 0.8805 & 3 \\
\hline 6 & 1PQ9 & Oxysterols receptor LXR-beta & 0.8675 & 3 \\
\hline 7 & 1Q8M & $\begin{array}{l}\text { Triggering receptor expressed on myeloid } \\
\text { cells 1 }\end{array}$ & 0.843 & 3 \\
\hline
\end{tabular}


Table 3. Target prediction results of the PDB database

\begin{tabular}{|c|c|c|c|c|}
\hline Rank & Ligand Structure & Ligand Name & PDB ID & Protein Name \\
\hline 1 & & $5 \mathrm{~K} 3$ & 4CUQ & $\begin{array}{l}\text { Bromodomain adjacent to } z \text { inc } \\
\text { finger domain protein } 2 \mathrm{~B}\end{array}$ \\
\hline 2 & & P18 & $4 \mathrm{~B} 55$ & $\begin{array}{c}\text { Arylamine N-acetyltransferase } \\
\text { Nat }\end{array}$ \\
\hline
\end{tabular}

\section{PDB protein database target screening results}

The similarity of ligand compounds was used to predict the target in the PDB protein database. Two potential targets were obtained by virtual filtering, and their PDB ID was 4CUQ and 4B55. The corresponding protein of $4 \mathrm{CUQ}$ is bromodomain adjacent to zinc finger domain protein $2 \mathrm{~B}$, which is related to the occurrence and development of Alzheimer's disease. The protein corresponding to 4B55 is arylamine $\mathrm{N}$-acetyltransferase $\mathrm{Nat}$, a protein target associated with cancer and inflammation. Therefore, the results of screening potential targets of compounds using the PDB protein database show that the compound may have potential anti-cancer and anti-inflammatory activities. The ligand information is shown in Table 3. 
Table 4

Target prediction results of ROCS

\begin{tabular}{|c|c|c|c|}
\hline Disease & ShapeTanimoto & $\begin{array}{l}\text { PDB } \\
\text { ID }\end{array}$ & Protein Name \\
\hline \multirow[t]{4}{*}{$A D$} & 0.8695 & $4 \mathrm{KZO}$ & $\begin{array}{l}\text { Phosphatidylinositol 4,5-bisphosphate 3-kinase } \\
\text { catalytic subunit gamma isoform }\end{array}$ \\
\hline & 0.8184 & $3 Z R L$ & Serine/threonine-protein kinase GSK3B \\
\hline & 0.8102 & $1 \mathrm{UNG}$ & Cyclin-dependent kinase 5 \\
\hline & 0.8066 & $3 F M J$ & Mitogen-activated protein kinase 14 \\
\hline \multirow[t]{12}{*}{ Cancer } & 0.9036 & $3 \mathrm{D} 9 \mathrm{Z}$ & Carbonic anhydrase 2 \\
\hline & 0.88 & 2YEI & Heat shock protein HSP 90-alpha \\
\hline & 0.8767 & $1 \mathrm{M} 9 \mathrm{M}$ & Nitric oxide synthase, endothelial \\
\hline & 0.8745 & 2QGW & Estrogen receptor \\
\hline & 0.8695 & $4 \mathrm{KZO}$ & $\begin{array}{l}\text { Phosphatidylinositol 4,5-bisphosphate 3-kinase } \\
\text { catalytic subunit gamma isoform }\end{array}$ \\
\hline & 0.8666 & 4EK4 & Cyclin-dependent kinase 2 \\
\hline & 0.8332 & $1 \mathrm{X} 7 \mathrm{~J}$ & Estrogen receptor beta \\
\hline & 0.833 & $3 F 16$ & Macrophage metalloelastase \\
\hline & 0.8296 & 2YM6 & Serine/threonine-protein kinase Chk1 \\
\hline & 0.824 & 3CQW & RAC-alphaserine/threonine-protein kinase \\
\hline & 0.8067 & $4 \mathrm{BDC}$ & Serine/threonine-protein kinase Chk2 \\
\hline & 0.8026 & 2JNP & Stromelysin-1 \\
\hline \multirow[t]{3}{*}{ Diabetes } & 0.8411 & $3 A 29$ & Fructose-1,6-bisphosphatase 1 \\
\hline & 0.824 & 3CQW & RAC-alpha serine/threonine-protein kinase \\
\hline & 0.8184 & $3 Z R L$ & Serine/threonine-protein kinase GSK3B \\
\hline \multirow[t]{4}{*}{ Inflammation } & 0.8695 & $4 \mathrm{KZO}$ & $\begin{array}{l}\text { Phosphatidylinositol 4,5-bisphosphate 3-kinase } \\
\text { catalytic subunit gamma isoform }\end{array}$ \\
\hline & 0.8325 & $1 \mathrm{RCT}$ & Purine nucleoside phosphorylase \\
\hline & 0.8066 & $3 \mathrm{FMJ}$ & Mitogen-activated protein kinase 14 \\
\hline & 0.8026 & 2JNP & Stromelysin-1 \\
\hline
\end{tabular}


ROCS is a fast tool for shape comparison. The ROCS server is used to predict the target, and the target is sorted by ShapeTanimoto value from high to low by default. The target proteins of the compound in Alzheimer's disease, cancer, diabetes, and inflammation were predicted in the ROCS server. Protein targets with ShapeTanimoto $>0.8$ were selected for further analysis. The prediction results are shown in Table 4 . In terms of Alzheimer's disease, 4 potential targets of the compound were found. In terms of cancer, 12 targets were predicted. In the case of diabetes, obtaining three targets that meet the requirements. In terms of inflammation, 4 targets meet the screening requirements. The target information obtained shows that the research potential of the compound in cancer is greater than that in the other three diseases.

Table 5

Docking binding energy of the compound and proligands with target proteins

\begin{tabular}{|llllll|}
\hline $\begin{array}{l}\text { PDB } \\
\text { ID }\end{array}$ & $\begin{array}{l}\text { Binding } \\
\text { energy(kcal/mol) } \\
\text { (Compound) }\end{array}$ & $\begin{array}{l}\text { Binding } \\
\text { energy(kcal/mol) } \\
\text { (Reference) }\end{array}$ & $\begin{array}{l}\text { PDB } \\
\text { ID }\end{array}$ & $\begin{array}{l}\text { Binding } \\
\text { energy(kcal/mol) } \\
\text { (Compound) }\end{array}$ & $\begin{array}{l}\text { Binding } \\
\text { energy(kcal/mol) } \\
\text { (Reference) }\end{array}$ \\
\hline 1M9M & -119.00 & -132.16 & 3FMJ & -73.95 & -87.39 \\
\hline 3F16 & -90.73 & -103.49 & 1W6F & -73.94 & -75.68 \\
\hline 4B55 & -90.50 & -61.05 & 4CUQ & -71.71 & -74.32 \\
\hline 3D9Z & -84.30 & -79.60 & 2YEI & -71.50 & -68.27 \\
\hline 3A29 & -83.61 & -110.85 & 1X7J & -71.31 & -105.61 \\
\hline 2JNP & -80.77 & -106.70 & 3CQW & -67.74 & -78.80 \\
\hline 1RCT & -78.96 & -116.33 & 1UNG & -67.00 & -82.54 \\
\hline 1KLT & -78.17 & -73.38 & 2QGW & -66.52 & -89.28 \\
\hline 4KZ0 & -77.62 & -93.44 & 3ZRL & -66.01 & -70.76 \\
\hline 1DX6 & -76.37 & -107.09 & 4EK4 & -65.52 & -85.34 \\
\hline 1SV3 & -76.19 & -69.42 & 4BDC & -63.72 & -84.43 \\
\hline 2YM6 & -76.03 & -89.44 & 1W8M & -62.68 & -56.89 \\
\hline 1PQ9 & -75.92 & -49.88 & 1Q8M & -56.56 & -73.41 \\
\hline
\end{tabular}

\section{Molecular docking results}

The iGEMDOCK software was used to molecularly dock the compound with the 26 target proteins obtained by the four target screening methods. The docking score is an important method to evaluate the binding effect between small molecule ligand and protein. The higher the absolute value of the docking score, the greater the binding effect of the small molecule ligand to the protein and the better the 
matching degree with the target cavity; otherwise, the worse. The docking energy is shown in Table 5. The results of docking showed that the binding energies of compound and target proprotein ligands docked with target proteins were compared. The results showed that the binding energy of the compound with oxysterols receptor LXR-beta, chymase, heat shock protein HSP 90-alpha, carbonic anhydrase 2, and arylamine $\mathrm{N}$-acetyltransferase Nat was higher than that of the proligand of the target protein. The binding energies of the compound with arylamine $\mathrm{N}$-acetyltransferase Nat and oxysterols receptor LXR-beta were the most different, which were $-29.45 \mathrm{kcal} / \mathrm{mol}$ and $-26.04 \mathrm{kcal} / \mathrm{mol}$, respectively. The results showed that the affinity of the compound to these target proteins was higher than that of the original ligands. Therefore, the compound may have potential applications in lipid transport, inflammation, cancer, and intestinal flora.

\section{Pharmacophore model construction results}

Based on the docking results, the pharmacophore model of the compound was constructed by using Liganscout program. The amino acid residues and forces that interact with ligand molecules in the complex were compared. The results show that the phenolic hydroxyl group in the compound is the key group and can participate in the interaction with amino acids. According to the comparison with the action mode of each protein co-crystallization compound, it is found that the phenolic hydroxyl groups in the co-crystallization compounds and the investigated compounds can participate in the receptor-ligand interaction. Besides, the mother nuclear benzene ring can also participate in the hydrophobic interaction. Therefore, phenolic hydroxyl groups can be used as the key pharmacophore characteristics of compounds. The comparison of the pharmacophore model is shown in Fig. 2.

\section{Conclusion}

Drug targets are the basis of drug screening, which is of source innovation significance for the creation of new drugs. Natural products are an important source of new drug creation. Identifying their targets can not only explore the applicability of drugs but provide possible new strategies for clinical prevention and treatment. The reverse target search of compounds can be carried out by using computer virtual screening technology, and the acting target proteins of natural products can be found more efficiently.

In this study, 26 potential targets were obtained by different screening methods. The diseases involved in these targets include Alzheimer's disease, oncology, diabetes, inflammation, cardiovascular disease. It is suggested that the compound may have a wide range of biological activities and have potential applications in anti-tumor, anti-inflammatory, anti-Alzheimer's disease, hypoglycemic, and prevention of cardiovascular diseases. Ligand compounds and target proligands were docked with target proteins one by one by molecular docking software. The results of docking showed that the binding affinity of the compound to LXR-beta, chymase, heat shock protein HSP 90-alpha, carbonic anhydrase 2, and arylamine $\mathrm{N}$-acetyltransferase Nat was higher than that of the original ligands, indicating that the compound has great application potential in anti-tumor, anti-inflammation, affecting intestinal flora and affecting human lipid transport. The pharmacophore models of molecular docking complexes were compared and analyzed by Ligandscout program. The results show that the phenolic hydroxyl group in the compound is 
the key group, which can participate in the interaction with amino acids. Besides, the mother nuclear benzene ring can also participate in the hydrophobic interaction. Although this study is based on virtual screening at the computer level, it provides a reference for further study on the anti-tumor and antiinflammatory mechanisms and pharmacodynamics of compounds.

\section{References}

1. Kirchweger, B. et al. In Silico Work flow for the Discovery of Natural Products Activating the G ProteinCoupled Bile Acid Receptor 1. Front Chem. 6, 242(2018).

2. Huang, H. et al. Reverse Screening Methods to Search for the Protein Targets of Chemopreventive Compounds. Front Chem. 6, 138(2018).

3. Szychowski, K. A. et al. Biological and anticancer properties of Inonotus obliquus extracts. Process Biochemistry. 73, 180-187(2018).

4. Jiang, S. et al. Inonotus obliquus polysaccharides induces apoptosis of lung cancer cells and alters energy metabolism via the LKB1/AMPK axis. Int J Biol Macromol. 151, 1277-1286(2020).

5 . Wang, J. et al. Antidiabetic activities of polysaccharides separated from Inonotus obliquus via the modulation of oxidative stress in mice with streptozotocin-induced diabetes. Plos One. 12, e0180476(2017).

6. Javed, S. et al. Inonotus obliquus attenuates histamine-induced microvascular inflammation. Plos One. 14, e0220776(2019).

7. Han, Y., Nan, S., Fan, J., Chen, Q. \& Zhang, Y. Inonotus obliquus polysaccharides protect against Alzheimer's disease by regulating Nrf2 signaling and exerting antioxidative and antiapoptotic effects. Int J Biol Macromol. 131, 769-778(2019).

8. Zhao, F. et al. Chemical constituents from Inonotus obliquus and their antitumor activities. $J$ Nat Med. 70, 721-30(2016).

9. Taji, S. et al. Lanostane-type triterpenoids from the sclerotia of Inonotus obliquus possessing antitumor promoting activity. Eur J Med Chem. 43, 2373-2379(2008).

10. Pandya, U., huldhaj, U. \& Sahay, N. S. Bioactive mushroom polysaccharides as antitumor: an overview. Nat Prod Res. 33, 2668-2680(2019).

11. Ying, Y. M. et al. Terpenoids with alpha-glucosidase inhibitory activity from the submerged culture of Inonotus obliquus. Phytochemistry. 108, 171-176(2014).

12. Ahn, S. et al. Anti-inflammatory activity of ginsenosides in LPS-stimulated RAW 264.7 cells. Science Bulletin. 60, 773-784(2015).

13. Ammar, O. In silico pharmacodynamics, toxicity profile and biological activities of the Saharan medicinal plant Limoniastrum feei. Brazilian Journal of Pharmaceutical Sciences. 53, e0061(2017).

14. Bahuguna, A. et al. Bisindolemethane derivatives as highly potent anticancer agents: Synthesis, medicinal activity evaluation, cell-based compound discovery, and computational target predictions. Comput Biol Med. 116, 103574(2020). 
15. Cereto-Massague, A. et al. Tools for in silico target fishing. Methods. 71, 98-103(2015).

16. Lo, Y. C., Senese, S., Damoiseaux, R. \& Torres, J. Z. 3D Chemical Similarity Networks for StructureBased Target Prediction and Scaffold Hopping. ACS Chem Biol. 11, 2244-2253(2016).

17. AbdulHameed, M. D. et al. Exploring polypharmacology using a ROCS-based target fishing approach. J Chem Inf Model. 52, 492-505(2012).

18. Meslamani, J. et al. Protein-ligand-based pharmacophores: generation and utility assessment in computational ligand profiling. J Chem Inf Model. 52, 943-955(2012).

19. Iheagwam, F. N., Ogunlana, O. O., Ogunlana, O. E., Isewon, I. \& Oyelade, J. Potential Anti-Cancer Flavonoids Isolated From Caesalpinia bonduc Young Twigs and Leaves: Molecular Docking and In Silico Studies. Bioinform Biol Insights. DOI 1177932218821371(2019).

20. Poli, G., Seidel, T. \& Langer, T. Conformational Sampling of Small Molecules With iCon: Performance Assessment in Comparison With OMEGA. Front Chem. 6, 229(2018).

\section{Figures}



\section{Figure 1}

Chemical structure of the compound 


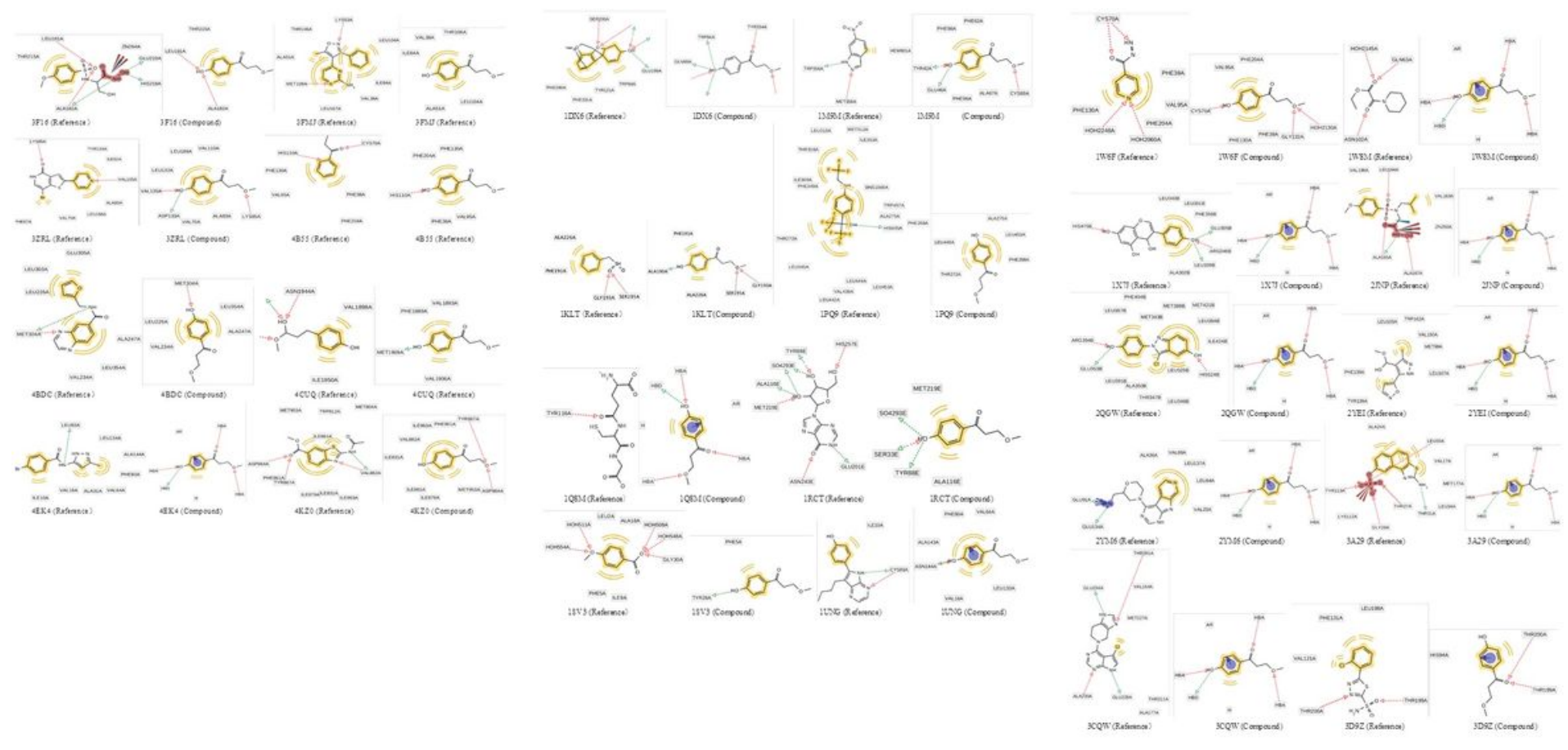

Figure 2

Comparison of pharmacophore models 